\title{
Radiologic Differences between Human Papillomavirus-Related and Human Papillomavirus-Unrelated Oropharyngeal Carcinoma on Diffusion-Weighted Imaging
}

\author{
Sarah Deschuymer Sandra Nuyts \\ Department of Oncology, KU Leuven - University of Leuven, and Experimental Radiotherapy, \\ University Hospitals Leuven, Leuven, Belgium
}

With great interest we have read the paper of Chan et al. [1] published online on March 1, 2017, with the title: "Radiologic Differences between Human Papillomavirus-Related and Human Papillomavirus-Unrelated Oropharyngeal Carcinoma on Diffusion-Weighted Imaging." It is the first published article that also compares the apparent diffusion coefficient (ADC) value of lymph node metastasis in human papillomavirus (HPV)+ and HPV- oropharyngeal tumors. The combination of the ADC values of the primary tumor and the lymph node metastasis results in a model with a very high area under the curve of 0.92 .

With the new TNM classification (8th edition), the stratification of HPV+ and HPV- tumors is not only the main prognostic factor in oropharyngeal carcinoma, it is also essential for tumor staging [2, 3]. At the moment, there is a lot of ongoing research on new treatments for HPV+ tumors [4]. So, the distinction between $\mathrm{HPV}+$ and HPV- tumors will become even more important and relevant in future clinical practice.

As the authors correctly state in the discussion, there is no consensus yet regarding the optimal HPV test: HPV DNA in situ hybridization, polymerase chain reaction analysis, or p16 immunohistochemistry [5]. We support the authors in their choice of using p16 immunohistochemistry as a surrogate measure of HPV status. It is an easily implementable test in routine clinical practice and has been used in previous large studies [5-7].

However, we have some questions regarding the methodology used in this paper concerning the differentiation between HPV+ and HPV- tumors. The authors state: "A HPV-positive status was defined as $>70 \%$ nuclear and cytoplasmic p16 positivity. Staining above 0 but $<70 \%$ was considered equivocal, and those cases were not included in the study." We completely agree with the cutoff of $>70 \%$ nuclear and cytoplasmic p16 positivity. This cutoff has been used in numerous previous studies [5, 6].

Though excluding all patients with staining above 0 but $<70 \%$ seems strange. In daily clinical practice, these patients would be considered HPV- patients. The HPV- patient group in this paper includes only patients who have $0 \%$ p 16 staining. Those 11 patients are a subgroup of a bigger group of patients who would generally be considered as HPV- (all patients with $<70 \%$ p16 staining). There is also no information in the article about the number of patients excluded based on p16 staining above 0 but $<70 \%$. The ADC results and the area under the curve of the model could be different if these patients were also included.

Sarah Deschuymer

Department of Oncology, KU Leuven - University of Leuven

Experimental Radiotherapy, University Hospitals Leuven

Herestraat 49, BE-3000 Leuven (Belgium)

E-Mail sarah.deschuymer@uzleuven.be 
Deschuymer and Nuyts: Letter to the Editor: Radiologic Differences between HPV-Related and HPV-Unrelated Oropharyngeal Carcinoma

\section{Disclosure Statement}

There is no conflict of interest to declare.

\section{References}

1 Chan MW, Higgins K, Enepekides D, Poon I, Symons SP, Moineddin R, Weinreb I, Shearkhani O, Chen A, Beelen J, Chan A, Maralani PJ: Radiologic differences between human papillomavirus-related and human papillomavirus-unrelated oropharyngeal carcinoma on diffusion-weighted imaging. ORL 2016;78:344-352.

2 Ang KK, Harris J, Wheeler R, Weber R, Rosenthal DI, Nguyen-Tan PF, et al: Human papillomavirus and survival of patients with oropharyngeal cancer. N Engl J Med 2010;363:24-35.

3 O'Sullivan B, Huang SH, Garden AS, Sturgis AM, Dahlstrom K, Lee N, et al: Development and validation of a staging system for HPV-related oropharyngeal cancer by the International Collaboration on Oropharyngeal Cancer Network for Staging (ICON-S): a multicentre cohort study. Lancet Oncol 2016;17:440-451.

4 Bhartia A, Burtness B: Human papillomavirus-associated oropharyngeal cancer: defining risk groups and clinical trials. J Clin Oncol 2015;33:3243-3250.

5 Larsen CG, Gyldenlove M, Jensen DH, Therkildsen MH, Kiss K, Horrild B, et al: Correlation between human papillomavirus and p16 overexpression in oropharyngeal tumours: a systematic review. Br J Cancer 2014; 110:1587-1594.

6 Lassen P, Overgaard J: Scoring and classification of oropharyngeal carcinoma based on HPV-related p16-expression. Radiother Oncol 2012;105:269-270.

7 Bentzen J, Toustrup K, Eriksen JG, Primdahl H, Andersen LJ, Overgaard J: Locally advanced head and neck cancer treated with accelerated radiotherapy, the hypoxic modifier nimorazole and weekly cisplatin. Results from the DAHANCA 18 phase II study. Acta Oncol 2015;54:1001-1007. 